\title{
Effects of Chlorella sp. on biological characteristics of the honey bee Apis mellifera
}

\author{
Tomáš JeHLiK ${ }^{1,2}$, Dalibor KodRíK ${ }^{1,2}$, Václav KRIŠTƯFeK ${ }^{3}$, Justina KoubovÁ ${ }^{1,2}$,

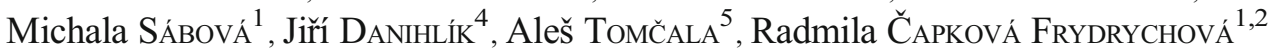 \\ ${ }^{1}$ Institute of Entomology, Czech Academy of Sciences, Biology Centre, Branišovská 31, 370 05, České Budějovice, \\ Czech Republic \\ ${ }^{2}$ Faculty of Science, University of South Bohemia, Branišovská 31, 370 05, České Budějovice, Czech Republic \\ ${ }^{3}$ Institute of Soil Biology, Czech Academy of Sciences, Biology Centre, Branišovská 31, 370 05, České Budějovice, \\ Czech Republic \\ ${ }^{4}$ Faculty of Science, Palacký University, Šlechtitelů 27, 783 71, Olomouc, Czech Republic \\ ${ }^{5}$ Institute of Parasitology, Czech Academy of Sciences, Biology Centre, Branišovská 31, 370 05, České Budějovice, \\ Czech Republic
}

Received 23 November 2018 - Revised 9 May 2019 - Accepted 24 June 2019

\begin{abstract}
We tested the effect of Chlorella sorokiniana, a green, unicellular, freshwater alga, provided as a food supplement on several biological characteristics of the honey bee Apis mellifera. Chlorella was applied as (1) a moisturized powder, (2) a sugar-water solution, or (3) mixed with honey-sugar candy. All three applications were well accepted by the bees. We observed a positive effect of Chlorella on colony development, and also on basic aspects of metabolism, such as increased fat deposition and vitellogenin transcript levels, and a decrease in TOR and InR2 transcript levels. The effect of Chlorella on other characteristics was lower (protein levels) or even null (total fat body mass, level of adipokinetic hormone). Application of Chlorella modulated the hypopharyngeal gland size, and the activity of basic digestive enzymes in the bee midgut. Our observations suggest that the nutritional composition of Chlorella might be an appropriate dietary supplement for honey bees.
\end{abstract}

\section{A. mellifera / chlorella / nutrients / longevity / AKH}

\section{INTRODUCTION}

The majority of flowering plants rely on insect pollination as a key ecosystem service, which is provided mostly by insects, especially bees. Honey bees and bumble bees also play a pivotal role in many agricultural cropping systems, pollinating

Electronic supplementary material The online version of this article (https://doi.org/10.1007/s13592-019-00670-3) contains supplementary material, which is available to authorized users.

Corresponding author: R. Čapková Frydrychová, radmila.frydrychova@hotmail.com Manuscript editor: James Nieh
$75 \%$ of agricultural crops (reviewed in Potts et al. 2010). The use of managed bees is essential, especially in large monoculture farming where a large amount of pollination services are required, in areas where populations of natural pollinators are reduced or absent, or for pollination of greenhouse grown crops. However, a gradual decline in honey bee populations worldwide during recent decades (e.g. up to $50 \%$ of European colonies) has raised serious concerns about the low availability pollination services in the future, posing a serious threat to crop yields (Brodschneider et al. 2018; Neumann and Carreck 2010).

Loss of natural environments and intensive agriculture strongly decrease the availability of 
pollen resources, which may lead to an improper amino acid composition in pollen intake, causing nutritional stress in honey bees and perhaps driving colony loss (Naug and Gibbs 2009). Pollen is a main source of proteins and is a limiting factor for proper development and health of a bee colony (DeGrandi-Hoffman et al. 2010).

When natural food sources are temporally inadequate or not available, appropriate nutrition supplemental foods, such as carbohydrate and protein supplements, are provided to honey bee colonies. Providing carbohydrate supplements, i.e. honey or cane or beet sugar syrup, usually stimulates queens to begin laying eggs; however, well-maintained egg laying also demands adequate pollen supply (Standifer et al. 1978). There are numerous commercially available pollen supplements based on brewer's yeast, soybean, mungbean, or chick pea that are well accepted by bees and provide the appropriate quality and quantity of nutrients for honey bee development and colony growth. However, none of these supplements are considered as a complete replacement for natural pollen.

Nutrition is a key factor in caste differentiation process and lifespan regulation in A. mellifera, activating a cross-talk among several signalling pathways. One factor of lifespan regulation is vitellogenin (Vg), a 118-kDa yolk protein precursor, synthesized in the fat body and transported by haemolymph into developing eggs, and in adult bees improving their nutrition status and health condition (Amdam and Omholt 2002; Havukainen et al. 2013). It is proposed that beneficial effects of vitellogenin may be also connected to its inhibitory action on juvenile hormone and insulin-like signalling, which are usually considered as pro-ageing factors in adult bees (Hsieh and Hsu 2011; Münch and Amdam 2010; Mutti et al. 2011). Nutrient availability also regulates activity of target-of-rapamycin (TOR) pathway. It is known that during larval development, the elevated activity of TOR leads to queen destiny; however, increased levels of TOR are associated with ageing at workers (Hsu et al. 2014; Patel et al. 2007). Insect metabolism is controlled by several neurohormones of which adipokinetic (AKHs) hormones play a key role. AKHs are pleiotropic in nature and many activities are associated with their metabolic role. These hormones also regulate starvation-induced foraging behaviour in Drosophila, enhance food intake and digestive processes in insect guts, and interact with the cellular and humoral immune system. Thus, AKHs are typical stress hormones that increase significantly when an insect encounters a stressor (for review, see Kodrík 2008; Kodrík et al. 2015), such that AKH levels are markers of stressful situations or stress intensity.

In this study, we tested the effect of Chlorella sorokiniana (referred to herein as Chlorella) as a food supplement for bees. Chlorella is a green, unicellular, freshwater alga that is used as a human food supplement as it provides biological and pharmacological health benefits. Chlorella is a rich source of proteins (60-75\%), fats (10-20\%, including omega-3 polyunsaturated fatty acids), vitamins, and minerals (min. 15\%), antioxidants (min. $1 \%$ ), and roughage ( $\min .5 \%$ ) (data obtained from the Centre Algatech, Institute of Microbiology CAS, Třeboň, Czech Republic). After providing a Chlorella diet during early spring, we observed a positive effect on colony development. We recorded beneficial metabolic changes, such increased fat deposition, increased vitellogenin transcript levels, and decreased TOR (target of rapamycin) and InR2 (insulin-like receptor like) transcript levels in adults. We found that Chlorella increased the size of hypopharyngeal glands. Our results suggest that the nutritional composition of Chlorella positively affected several aspects of honey bee biology.

\section{MATERIALS AND METHODS}

\subsection{Bees}

The experiments were conducted with the honey bee Apis mellifera carnica. Bees were kept in two apiaries: in experimental hives of the Biology Centre in České Budějovice (48 58' 31.924" N, $14^{\circ} 26^{\prime} 44.671$ "E; $390 \mathrm{~m}$ ), and at Henčov near Jihlava $\left(49^{\circ} 24^{\prime} 46.308^{\prime \prime} \mathrm{N}, 15^{\circ} 38^{\prime} 8.525^{\prime \prime} \mathrm{E}\right.$; $530 \mathrm{~m}$ ) both in the Czech Republic, and around 40 colonies from each apiary were used for the experiments. The bees were maintained according to standard bee-keeping techniques. Bee samples 
were collected at the very end of each experimental feeding (see below).

\subsection{Chlorella application and bee sampling}

In the hive experiments, the bees were supplied with autotrophic alga Chlorella sp. Chlorella was provided by the Centre Algatech, Institute of Microbiology CAS, Třeboň, Czech Republic. Chlorella was applied either as the moisturized Chlorella powder (designated as Chlorella powder, provided in 5 doses for each experiment where the dose was $20 \mathrm{~g}$ ), or as $5 \mathrm{~kg}$ of mixture of Chlorella powder with sugar, honey, and water (0.5:10:1:1.5), designated as Chlorella candy and provided in a single dose for each experiment, or $0.5 \%(w / v)$ Chlorella powder in a solution of sugar and water (3:2), designated as Chlorella solution and provided in 4 doses for each experiment where the dose was 51 . As controls, included in each Chlorella experiment, we used a honeysugar candy (sugar, honey, and water; 10:1:1.5) or sugar-water solution (3:2). The Chlorella application and bee sampling/evaluation the size of the brood population were performed within four periods. (1) In spring period 2017, Chlorella powder or Chlorella candy was applied during February and March, and the size of the brood population was evaluated in March (at Henčov locality). (2) In summer period 2017, Chlorella solution was applied during July and August, and sampling was performed in September 2017 and January 2018 (at Henčov locality; experiments to evaluate adipokinetic hormone and nutrient levels at workers and larvae). (3) In winter period 2018, Chlorella powder was applied during January 2018 and February 2018, and prior to the feeding, frames with all pollen supply were removed from the Chlorella experimental hives. The size of the brood population was evaluated in March (at České Budějovice locality). (4) In summer period 2018, Chlorella solution was applied during July, and sampling was performed in August (at České Budějovice locality; experiments to evaluate hypopharyngeal gland size and endocrine markers at workers). Colonies chosen for the experiments were all of approximately equal strength. All colonies were in hives with 2 supers and 20 frames $(39 \times 24 \mathrm{~cm})$ in total. The food was placed on the top bars of hives, and bees were fed ad libitum. Bee larvae and adult workers were collected, frozen in liquid nitrogen, and stored at $-20^{\circ} \mathrm{C}$ until used.

With exception of overwintering generation sampled in January 2018, we used 6-day-old workers for all laboratory experiments. Before the experiments, we marked freshly emerged workers by enamel paint on their thorax, left them in their colonies, and after 5 days, we used them for the experiments. When worked with larvae, we used 6-day-old larvae.

For determination of enzyme activity and $\mathrm{AKH}$ and nutrient levels, bees at both Chlorella and control group were collected from 3 to 4 colonies, and the same individuals were used for quantitation of the tested parameters.

\subsection{Cage experiments}

The cage experiments were used to test transcript levels of endocrine markers. Bees were collected within $24 \mathrm{~h}$ of emergence from a naturally mated queen colonies and were placed into small cages (250-ml plastic cup with ventilation and feeding holes) in groups of 25 individuals. Each cage was equipped with a feeder made out of a 20-ml syringe with a $0.5 \%(w / v)$ Chlorella mixture in $50 \%(w / v)$ sucrose or in $50 \%(w / v)$ sucrose as a control. The bees were caged at $34{ }^{\circ} \mathrm{C}$ for 7 days. Five independent experiments during June and July 2017 were performed. Each experiment was run in five replicates for both Chlorella and control group, and bees originated from one colony.

\subsection{Spectrophotometric determination of nutrients}

We monitored the effect of Chlorella diet on nutrient levels (both nutrient concentration and total nutrient content). Levels of proteins, lipids, and glycogen were determined in the bee fat body dissected from experimental individuals under the dissecting microscope and Ringer saline. The weighted fat bodies were homogenized in a corresponding buffer (see below), and the same extracts immediately processed for determination of protein, glycogen, and lipid levels. 
- Protein determination - fat bodies were individually homogenized in $0.2 \mathrm{M}$ Tris- $\mathrm{HCl}$ buffer, $\mathrm{pH} 7.8$, and protein quantification was done by the Bicinchoninic Acid Protein Assay Kit (Sigma-Aldrich) (Stoscheck 1990).

- Lipid determination - fat bodies were individually homogenized in chloroform:methanol (2:1) mixture, and the total lipid determination was done by the sulpho-phosho-vanillin method according to Kodrík et al. (2000). The optical densities at $546 \mathrm{~nm}$, measured in a spectrophotometer (UV 1601 Shimadzu, Japan), were converted to microgram lipids with the aid of a calibration curve based on known doses of oleic acid.

- Glycogen determination - fat bodies were homogenized in $70 \%$ ethanol, and glycogen levels were determined as described by Socha et al. (2004). For the calibration curve, known doses of glucose were used.

\subsection{Enzyme activity determination}

Activities of proteases, amylases, and lipases were determined in the midgut dissected from the adults using dissecting microscope and Ringer saline.

- Protease assay - the protease activity was assessed with the resorufin-casein kit (Roche). The results were expressed in units of proteolytic activity per milligram of the fresh organ weight. This unit (U) was defined as the amount of enzyme (mg) that caused an increase in optical density by 0.1 per min in $1 \mathrm{ml}$ of the reaction mixture.

- Amylase assay - the assay was performed with 3,5-dinitrosalicylic acid reagent (DNS) according to Kodrík et al. (2012). The enzyme activity was calculated in micromole maltose per milligram of the fresh organ weight.

- Lipase assay - the lipase activity was assessed with 4-methylumbelliferyl butyrate (4-MU butyrate) according to Kodrík et al. (2012). The results were expressed in nanomoles of $4-\mathrm{MU} / \mathrm{min} / \mathrm{mg}$ of fresh organ weight.

\subsection{Mass spectrometry determination of fatty acids}

The fat body lipids were extracted by a chloroform-methanol mixture as described above. The extracts were dissolved in methanol and mixed with internal standard phosphatidylglycerol (PG) 17:0/17:0 (Sigma-Aldrich). High-performance liquid chromatography (HPLC), combined with electrospray ionization tandem mass spectrometry (ESI-MS/MS), was used for analyses of the extracted lipids. A linear ion trap LTQ-XL mass spectrometer (Thermo Scientific, San Jose, CA, USA) coupled to Allegro ternary HPLC system equipped with an Accela autosampler and a thermostat chamber (all from Thermo, San Jose, CA, USA) were employed. For details of the analysis, see Schneedorferová et al. (2015). The data from HPLC ESI-MS/MS analyses were acquired and processed using Xcalibur software version 2.1 (Thermo Fisher Scientific, San Jose, CA, USA). The identification of particular lipid class and species, and fatty acids was achieved by measured mass, retention time, and fragmentation pattern.

\subsection{Adipokinetic hormone extraction and determination}

The central nervous system (CNS) containing the brain with corpora cardiaca was dissected from the adult head in the Ringer saline. The peptide fraction containing AKH was extracted using $80 \%$ methanol, the solution was evaporated, and the resulting pellet was used for determination of AKH content in CNS by a common direct ELISA. The primary rabbit antibodies were raised commercially against the A. mellifera AKH Schgr-AKH-II (pGlu-Leu-Asn-Phe-Ser-Thr-GlyTrp- $\mathrm{NH}_{2}$ ) (Marchal et al. 2018), and its dilution 1:1000 used in the ELISA procedure; to exclude a possible cross-reactivity, the corresponding preimmune serum was used as well. Swine anti rabbit IgG labelled with horse radish peroxidase (SwAR/HRP - LabNed) (dilution 1:2000) was used as a secondary antibody. Finally, the ELISA substrate 3,3',5,5'-tetramethylbenzidine (SigmaAldrich) was used to visualize the reaction. The absorbance values were determined in a microtiter plate reader at $450 \mathrm{~nm}$. 


\subsection{Monitoring brood rearing}

The amount of capped broods was assessed using a square grid consisting of 24 squares, each with an area of $35 \mathrm{~cm}^{2}$. The grids were placed over the brood frames, and the area of the capped brood was assessed. The brood measurements were performed after 42 days of supplemental feeding.

\subsection{Hypopharyngeal gland measurements}

The hypopharyngeal gland measurement was performed at six-day-old workers collected from hives after application of Chlorella (see above). The dissected hypopharyngeal glands were placed in a droplet of ice-cold Ringer's solution and recorded under stereo-microscope with an Olympus SZX 12 camera. The analysis of hypopharyngeal gland acini was performed using the Adobe Photoshop 11.0.2 pixel counting routine by measuring the areas of five randomly selected acini cells. Thus, 25 acini cells were examined for each colony for a total of 125 acini cells per treatment.

\subsection{RNA isolation, cDNA synthesis, and quantitative real-time PCR}

Total RNA samples were prepared from the whole heads of individual worker bees with a HybridR kit (GeneAll). The cDNA was prepared using SMARTscribe reverse transcriptase (Clontech) with $1 \mu \mathrm{g}$ total RNA primed with oligo (dT). Transcript levels of tested genes were measured using quantitative realtime PCR (qRT-PCR) on a Light Cycler CFX96 BioRad real-time PCR system using SYBR Premix Ex Taq ${ }^{\text {TM }}$ II (Takara). Threshold cycle values $(\mathrm{Ct})$ were normalized against Rpl13A and actin, and $\Delta \Delta \mathrm{Ct}$ method with correction for amplification efficiency was used to calculate levels of targets. When we measured transcript levels in our pilot experiments, data were normalized to both internal controls. As no significant differences were found between the data resulting from both normalization, in further experiments, the normalization was performed using Rpl13A only.
Sequences of primers to target genes ( $V g$, vitellogenin; TOR, target of rapamycin, InR2, insulin-like receptor like; JHE, juvenile hormone esterase) are shown in Table S1.

\subsection{Statistical analysis}

Statistical analyses were done using GraphPad Prism 6.0 (GraphPad Software, San Diego, CA, USA) by one-way ANOVA followed by Tukey's multiple comparison test, or by Student's $t$ test. The type of test used in individual analysis and number of replicates are specified in the figure legends. The bars in graphs represent the mean \pm SD.

\section{RESULTS}

\subsection{Chlorella stimulated brood rearing during early spring}

First, we tested effect of Chlorella, provided either as Chlorella powder or Chlorella candy, on brood rearing activity. Usually, bees utilized all amount of Chlorella food we provided to the tested colonies. We found that the addition of Chlorella to bee diets stimulated brood production during early spring (Figure 1). In the first experiment (performed in March 2017 in the apiary at Henčov), the control colonies showed $0.9 \mathrm{dm}^{2}$ of brood, whereas colonies fed with the moisturized Chlorella powder and Chlorella candy showed $13 \mathrm{dm}^{2}$ and $9 \mathrm{dm}^{2}$ of the capped brood, respectively (Figure 1a). An increase in brood rearing, although less profound, was also found in the subsequent experiment (performed in March 2018 in the apiary in České Budějovice) where all frames with all pollen supply were removed from the experimental hives prior to Chlorella powder feeding. Here, a roughly twofold increase was observed in the Chlorella colonies compared to the control ones (Figure 1b). No developmental defects or increased mortality upon Chlorella treatment was observed in any of the experiments. 

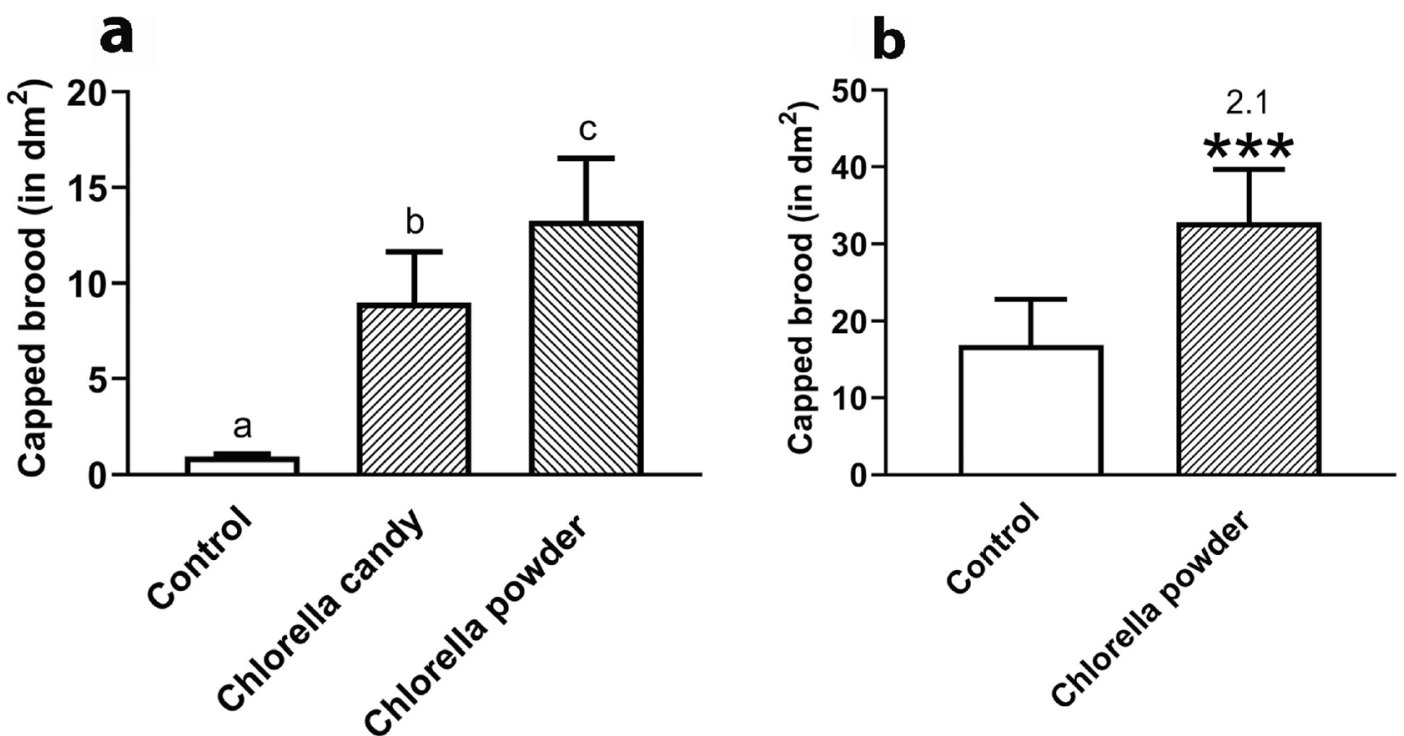

Figure 1. The effect of Chlorella on brood production. a Brood rearing evaluated during spring 2017 after Chlorella application. One-way ANOVA and Tukey's post hoc tests ( $p<5 \%$, indicated by different letters); $n=20-25$, where " $n$ " is the number of colonies for each tested group. b Brood rearing evaluated during spring 2018 when frames with pollen supply were removed from the hive prior to Chlorella powder application. Student's $t$ test $(* * * p<0.1 \%$; the average fold change is indicated by the number above the column; $n=20-25$, where " $n$ " is the number of colonies for each tested group).

\subsection{Effects of Chlorella on nutrient levels in fat body and on enzyme activity in midgut}

We monitored effect of Chlorella diet on fat body mass and nutrient levels in late summer larvae and subsequent winter generation of workers. Although there was no effect of Chlorella on the fat body mass (Figures $2 \mathrm{a}$ and $3 \mathrm{a}$ ), it changed nutrient concentrations. In larvae, concentrations of fat body proteins (Figure $2 b$ ) and lipids (Figure 2c) increased significantly (1.2-fold and 5.6-fold, respectively). Conversely, glycogen level was significantly reduced (3.8-fold) (Figure 2d). In winter adults we found no change in the concentration of fat body proteins (Figure $3 b$ ), but we observed a stimulatory effect on lipids (1.7-fold increase) (Figure 3c) and an inhibitory effect on glycogen levels (1.5-fold decrease) (Figure 3d). However, the effect of Chlorella on lipid and glycogen concentration in the fat bodies of adults was lower than that in larvae (compare Figure 2 vs. Figure 3).
We also compared the total amount of nutrients and fat body mass between larvae and adults (Figure 4). Generally, the larval fat body was much bigger than the adult one (Figure 4a). Furthermore, the amount of fat body proteins and lipids was significantly higher, and the amount of glycogen significantly lower, in larvae than in adult workers (Figure 4b, c, d). Interestingly, differences in lipids and glycogen levels (in $\mu$ g per organ) between controls and Chlorella groups were greater in larvae than in adult workers (control larvae vs. Chlorella larvae compared to control workers vs. Chlorella workers): within lipids (Figure 4c) 2.5-fold for larvae and 1.7-fold for adult workers; within glycogen (Figure 4d) 4.1fold for larvae and 1.5-fold for adult workers. This suggests a greater effect of Chlorella on larvae than on adults.

The next question was whether lipids in Chlorella groups were of the same structural quality as those in control groups. We tested the larval fat body as this showed the greatest response to Chlorella. The HPLC/ESI-MS analysis identified 

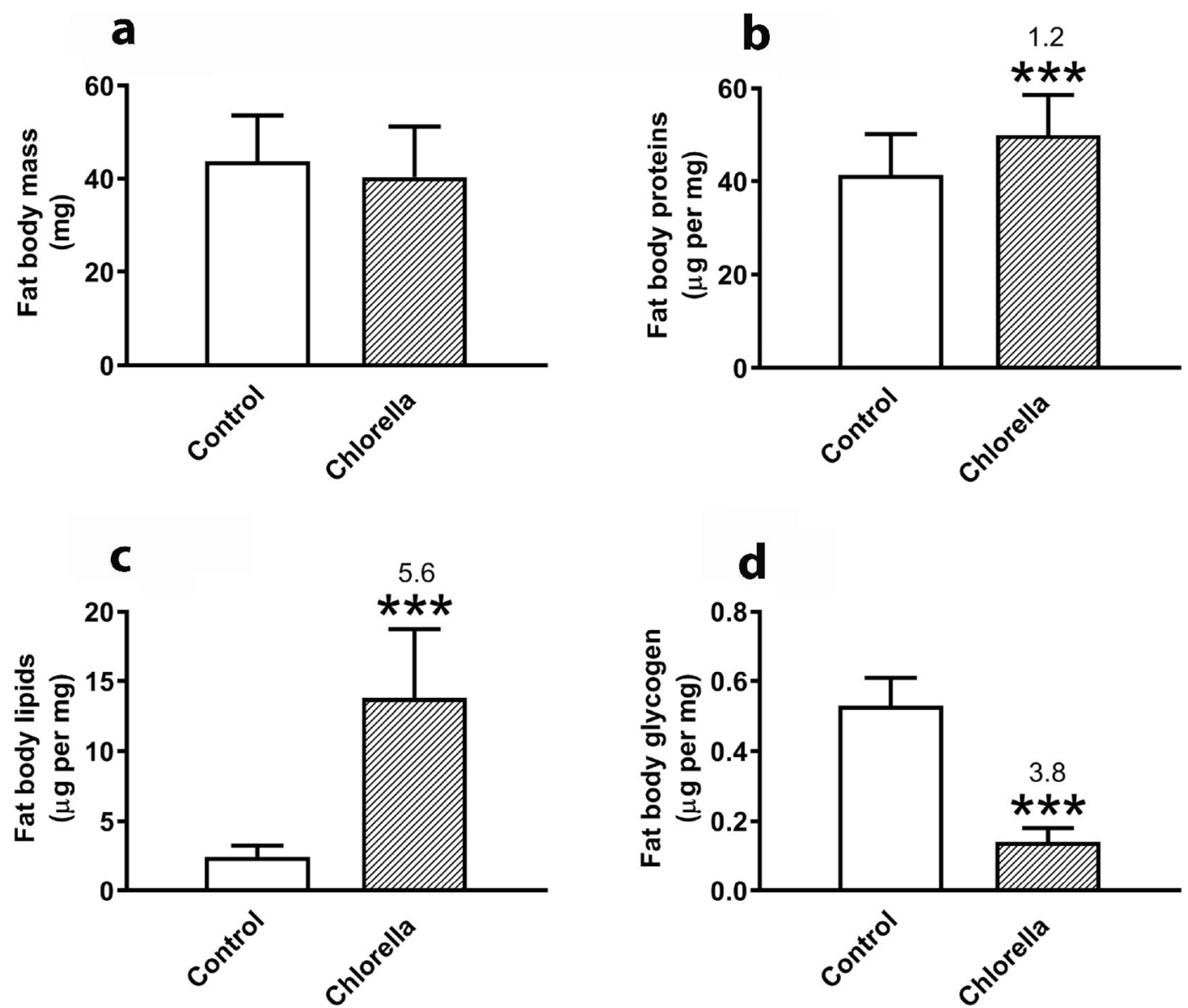

Figure 2. The effect of Chlorella on nutrient concentration in the larval fat body: total fat body mass (a), and levels of proteins (b), lipids (c), and glycogen (d). Student's $t$ test $(* * * p<0.1 \%$; the average fold change is indicated by the number above the column; $n=20-25$, where " $n$ " is the number of individuals for each tested group).

numerous glycerolipid species (data not shown), mostly triacylglycerols (TGs). Analysis of fatty acids (FAs) in these TGs revealed about 20 different FAs containing 12-26 carbons (data not shown). Nevertheless, eight FAs, which are common in insect lipids (Schneider and Dorn 1994), represented about $96 \%$ of all analysed fat body lipids (data not shown). Chlorella did not substantially affect the FA composition of analysed lipids (Fig. S1); the only significant difference between the Chlorella and control groups was in linoleic acid (18:3), which is rather minor in bee lipids.

Chlorella appeared to affect midgut activities. After receiving Chlorella, the midgut mass of winter workers increased significantly about 1.5 - fold (Figure 5a), and the activity of proteases (Figure 5b), lipases (Figure 5c), and amylases (Figure 5d) was reduced 1.5-fold, 2.9-fold, and 1.2-fold, respectively. The lower levels of enzymatic activity suggested easier digestibility of Chlorella nutrients.

\subsection{Effects of Chlorella diet on hypopharyngeal gland size}

To investigate whether Chlorella consumption has an impact on size of hypopharyngeal glands, we measured the size of acini cells of hypopharyngeal glands of young workers sampled from tested colonies, and we found that bees that 

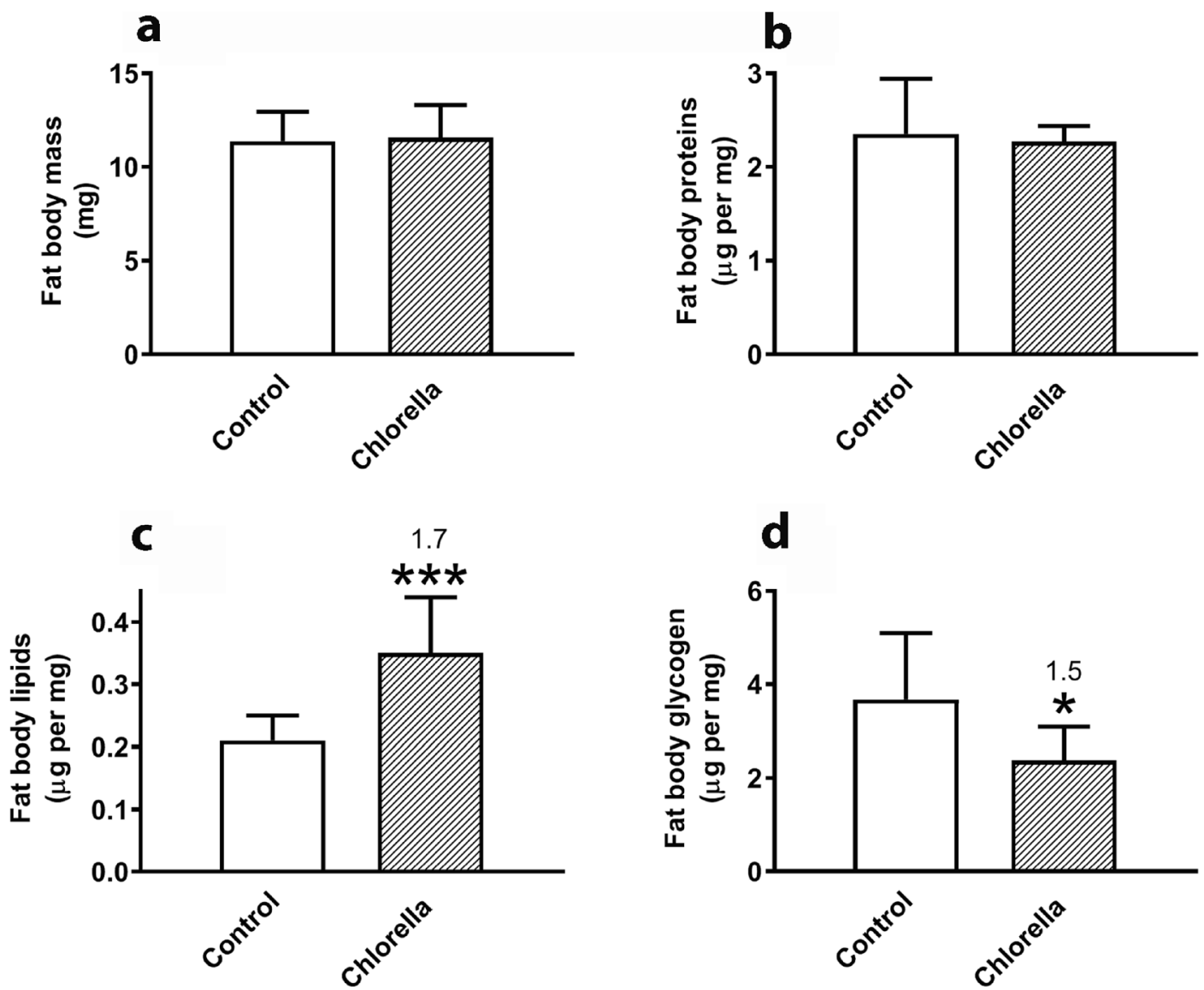

Figure 3. The effect of Chlorella on the nutrient concentration in adult worker fat body: total fat body mass (a), and levels of proteins (b), lipids (c), and glycogen (d). Student's $t$ test ${ }^{*} p<5 \%,{ }^{* *} p<0.1 \%$; the average fold change is indicated by the number above the column; $n=9-10$, where " $n$ " is the number of individuals for each tested group).

received Chlorella had a larger hypopharyngeal gland acinus $\left(0.02 \mathrm{~mm}^{2}\right)$ compared to control bees $\left(0.015 \mathrm{~mm}^{2}\right)$ (Figure 6).

\subsection{Chlorella-supplemented diet stimulated vitellogenin transcript level and decreases TOR and InR2 transcript levels}

Next, we evaluated transcript levels of endocrine markers that are involved in signalling pathways integrating information about nutrient availability (Mutti et al. 2011; Patel et al. 2007; Wang et al. 2014). Our results revealed that $V g$ transcript levels were stimulated by Chlorella (Figure 7). We found a significant 4.1-fold and 5.1-fold increase in $V g$ transcript levels in the hive and cage experiments, respectively. We tested the transcript levels of $J H E$, an enzyme that catalyses the hydrolysis of juvenile hormone. However, no consistent effects of Chlorella on JHE transcript levels were observed (data not shown). In the Chlorella groups during the hive experiments, we found a statistically significant decrease in transcript levels of InR2 (1.37-fold difference) and TOR (1.34fold difference). Also, statistically significant decrease in TOR was observed in the cage experiments (1.8-fold difference) (Figure 7). 

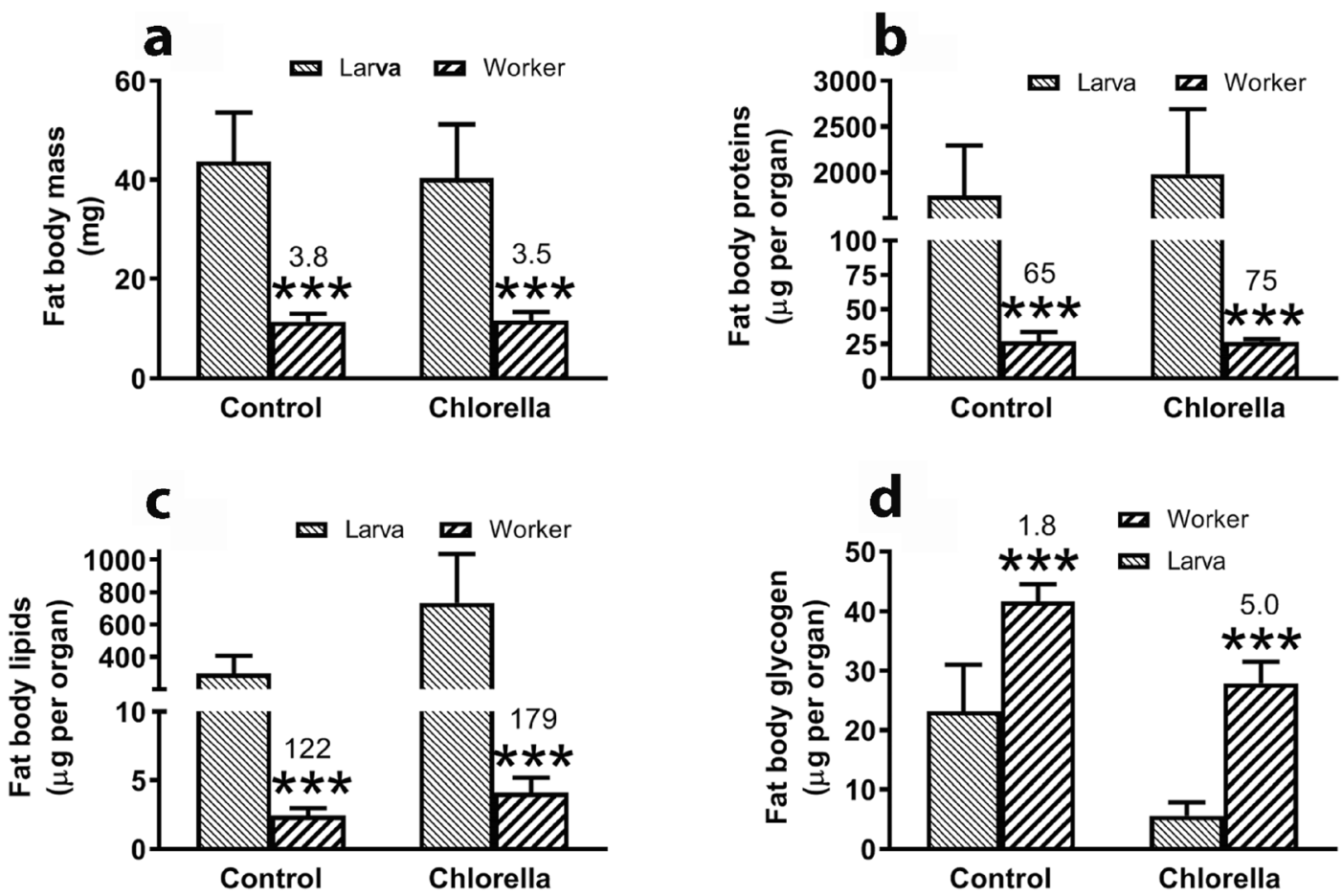

Figure 4. The effect of Chlorella on the total amount of nutrients in the fat body of late summer larvae and subsequent overwintering workers: total fat body mass (a), and amount ( $\mu \mathrm{g}$ per organ) of proteins (b), lipids (c), and glycogen (d). Chlorella was provided to colonies in summer 2017 and in winter 2018. The tested individuals were collected at the very end of each feeding period. Student's $t$ test $(* * * p<0.1 \%$; the average fold change is indicated by the number above the column; $n=9-25$, where " $n$ " is the number of individuals for each tested group).

\subsection{Effect of Chlorella diet on adipokinetic hormone level}

We measured levels of adipokinetic hormone, which plays a key role in insect energy metabolism. The primary rabbit antibody was raised against the A. mellifera AKH - Schgr-AKH-II (Schgr-AKH-II is AKH isoform originally identified in Schistocerca gregaria), and reactivity of the antibody was tested with the bee CNS extracts as well as with the synthetic Schgr-AKH-II and Manse-AKH (Manse-AKH is AKH isoform originally identified in Manduca sexta). The antibody reacted positively with the bee CNS extracts and the synthetic Schgr-AKH-II, no reactivity was found with synthetic Manse-AKH or with preimmune serum (data not shown), which supports recent suggestion that Schgr-AKH-II is intrinsic honey bee AKH (Marchal et al. 2018; see Discussion for details). Further, Chlorella had no effect on the level of Schgr-AKH-II in the CNS of adult workers (Fig. S2), suggesting that there was no nutritional stress from Chlorella; thus, Chlorella can satisfactorily fulfil the nutritional requirements of bees.

\section{DISCUSSION}

Honey bee nutrition depends on the food reserves stored in the hive. While nectar and honeydew are the main sources of sugars, pollen is the key source of proteins (24\%), lipids (5\%), essential amino acids, fibres, pectins, and vitamins. Variation in the availability of pollen resources strongly affects honey bee health (Di Pasquale et al. 2013); thus, a shortage or poor quality of pollen leads to nutritional stress, which might be a significant driver of colony decline (Naug and Gibbs 2009). There have been many attempts to use artificial pollen supplements or even pollen 

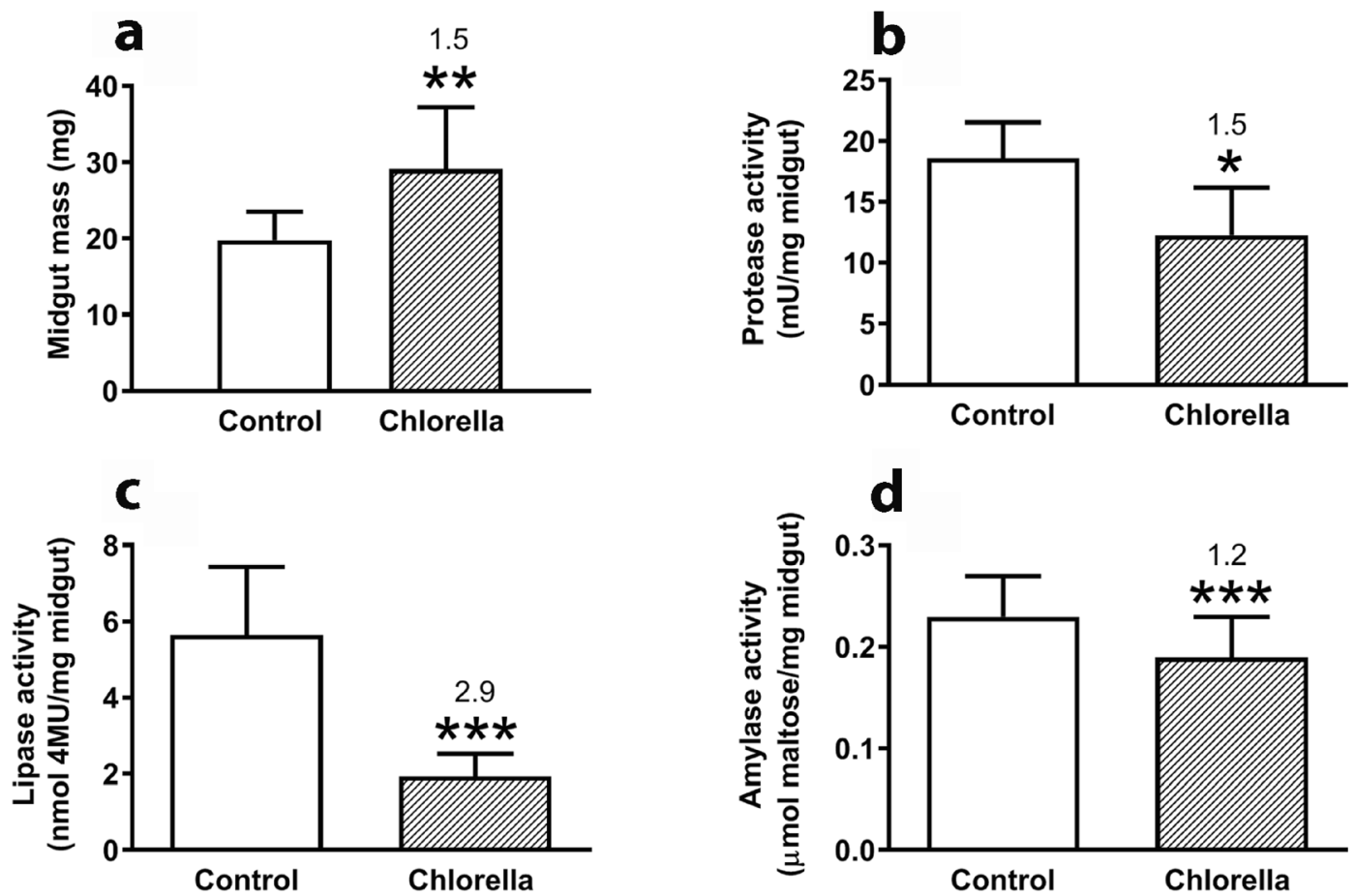

Figure 5. The effect of Chlorella on enzyme activity in adult worker midguts: total midgut mass (a), and activity of proteases (b), amylases (c), and lipases (d). Student's $t$ test $\left({ }^{*} p<5 \%, * * p<1 \%,{ }^{* * *} p<0.1 \%\right.$; the average fold change is indicated by the number above the column; $n=9-10$, where " $n$ " is the number of individuals for each tested group).

substitutes (Standifer et al. 1978), including algal proteins (Eremia et al. 2013). Such food is usually well accepted by bees and provides them with

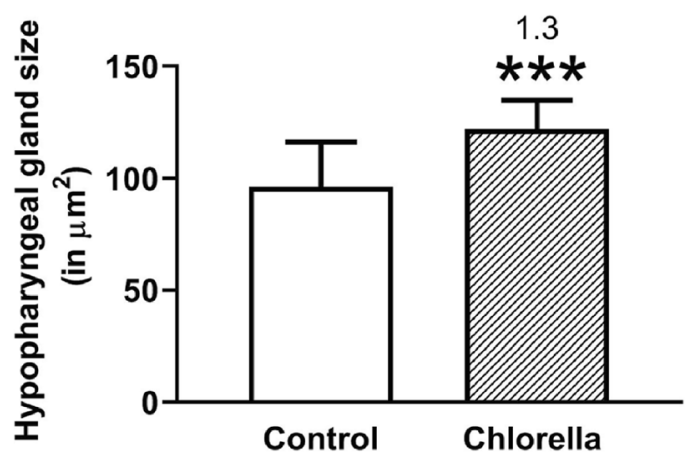

Figure 6. The effect of Chlorella on the hypopharyngeal gland size in adult workers. Student's $t$ test $(* * * p<0.1 \%$; the average fold change is indicated by the number above the column; $n=25$ ), where " $n$ " is the number of individuals for each tested group). quality nourishment, which results in a significant enlargement of the bee colony and honey production. However, very little is known about the effect of such supplements on bee nutritional status, development, and physiology. Chlorella provides a protein-rich diet and is also well accepted by bees. Chlorella supplements have been shown to stimulate growth of the bee colony and development of hypopharyngeal glands in nurse bees. Hypopharyngeal glands in young bees produce and secrete royal jelly, the food provided by nurses to the brood and queen (Winston 1987). The size of the glands can be limited by pollen intake, and pollen deprivation results in small hypopharyngeal glands leading to insufficient larval development (Omar et al. 2017; Peng et al. 2012).

Chlorella supplements can be beneficial to bee metabolism. We observed an increased concentration of lipids as energy reserve both in the bee larvae (mainly) and bee workers (mildly) after 

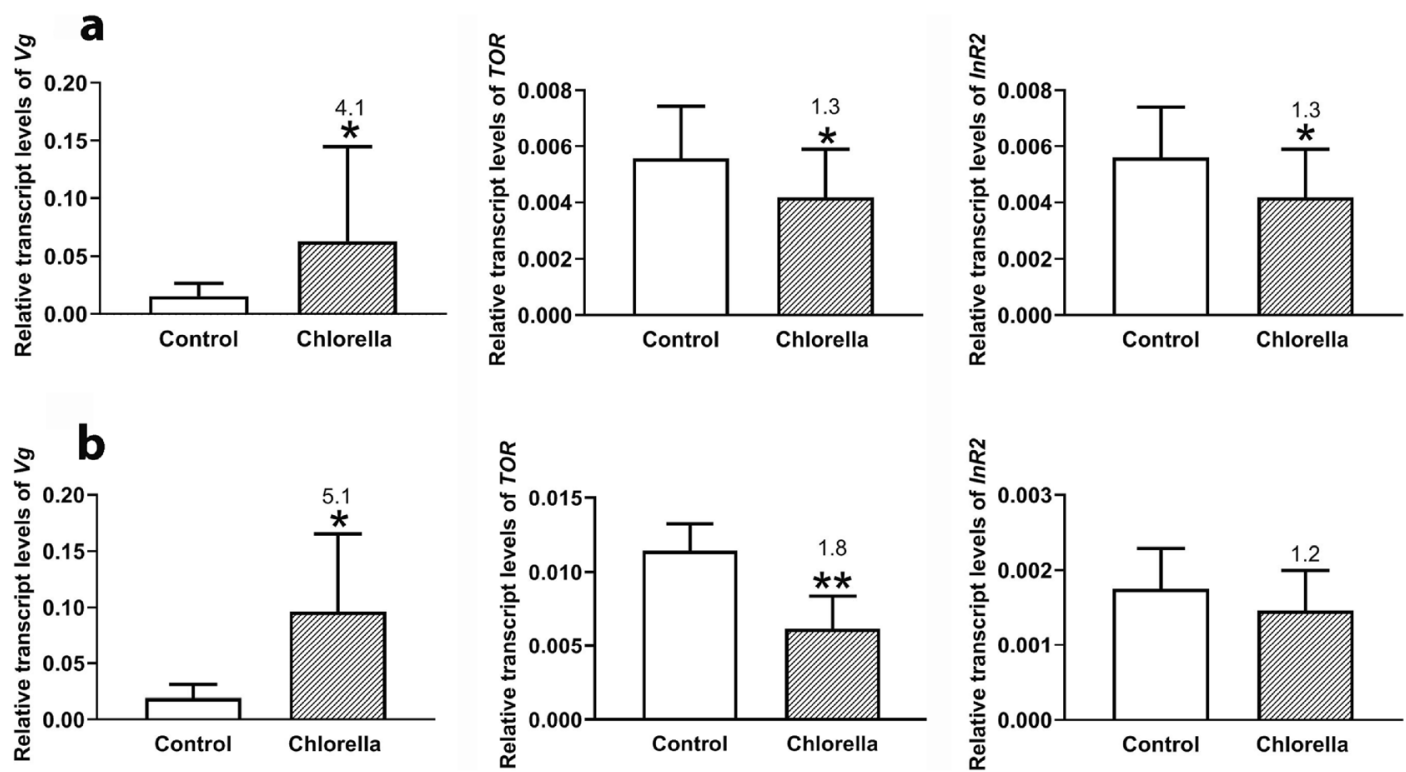

Figure 7. The effect of Chlorella on the relative transcript levels of tested genes in adult workers. Chlorella was applied in hive experiments (a) and in cage experiments (b). Student's $t$ test $\left(* p<5 \%\right.$, ${ }^{* *} p<1 \%$; the average fold change is indicated by the number above the column; $n=5$ ), where " $n$ " is the number of individuals for each tested group).

Chlorella feeding. Positive effects of Chlorella were observed predominantly in larvae, as evidenced by the ratio of total fat body lipids in larvae and adults.

Triacylglycerols (TGs) are essential components of insect fat bodies, representing about $90 \%$ of stored lipids (Arrese and Wells 1994). Several dozen FAs have been identified in insects; however, just eight to nine represent the majority. These FAs possess $12-18$ carbon atoms and include saturated, monounsaturated, and polyunsaturated FAs (Downer 1985; Schneider and Dorn 1994). We identified 20 different FAs in the fat body TGs of bee larvae; the majority (96\%) represented by eight FAs. These results are in accord with those published previously (Avni et al. 2014). Our results showed similar proportions of FAs in larvae fed Chlorella; the only difference being that linolenic acid increased about 1.7 -fold compared to the controls. It should also be noted that the fat body of bee larvae contained a relative high proportion of unusual FAs with an odd number of carbon atoms - saturated margaric acid (17:0). Besides being important nutrients, the
FAs may also play a role in bee immunity. For example, linoleic, linolenic, and myristic acids inhibit the growth of some bacteria, including the causative agent of American foulbrood, Paenibacillus larvae, a fatal infection of bee larvae (DeGrandi-Hoffman et al. 2018).

Undoubtedly, carbohydrates play an important role in bee metabolism and are present in high proportions in natural bee food. Surprisingly, Chlorella decreased glycogen concentration both in larval and adult fat bodies and reduced total glycogen in the fat body. Unlike proteins and lipids, the concentration of glycogen was higher in adult worker than larval fat bodies, and this difference was greater in the Chlorella groups. Further, the amount of glycogen in adult worker fat bodies was about 17 times higher than lipids, while glycogen was about 13 times lower than lipids. Chlorella increased this difference in larvae (from 13 to 131 ), but reduced it in workers (from 17 to 7). We concluded that the higher glycogen levels in adult workers reflected their more active way of life with 
access to an available source of energy, and the higher lipid levels in larvae indicated energy reserves that were more suitable for future larval development. Chlorella affected the energy management of bees, and the effect was greater in larvae than in adults. Further research is needed to explain this phenomenon.

In this study, we found no increase in $\mathrm{AKH}$ levels in the bee CNS after Chlorella, which suggested Chlorella did not disturb metabolic homeostasis or cause metabolic stress. It should be noted that identification of the honey bee AKH has been uncertain for a long time. Lorenz et al. (1999, 2001) identified the Manse-AKH in the bee CNS. Later, Veenstra et al. (2012) suggested the absence of any AKH expression in bees due to a second TATA box in the promoter of AKH gene producing mRNA that is unable to release final AKH. Recently, Marchal et al. (2018) demonstrated Schgr-AKH-II in the honey bee. It is difficult to collate these observations, but the discrepancies might be explained by complicated bee biology and possible fluctuations in AKH level during bee development (Lorenz et al. 1999). In any case, our antibody did not respond to synthetic Manse$\mathrm{AKH}$ or pre-immune serum, but recognized well the synthetic Schgr-AKH-II and reacted properly with the bee CNS extracts.

Proper functioning of the digestive system is a prerequisite for health and development in all animals. The highest enzyme activities in the bee gut are concentrated in the midgut. We found that Chlorella significantly increased midgut mass in adult workers. Conversely, the activity of midgut proteases, amylases, and lipases appeared to decline after Chlorella feeding. This might have reflected the ease of digestibility of Chlorella nutrients, which concurs with other favourable effects of Chlorella on bee characteristics.

We also tested whether Chlorella affected the transcript levels of genes that are related to lifespan and caste determination in bees. Although the exact mechanisms underlying lifespan in honey bees are unknown, nutrition is a crucial factor in the regulation of the caste differentiation process and lifespan in
A. mellifera. The caste differentiation process is mediated through several signalling pathways, including $\mathrm{Vg}$ production. $\mathrm{Vg}$ production is stimulated by a protein-rich diet, and elevated levels of $\mathrm{Vg}$ are associated with prolonged longevity (Aurori et al. 2014; Havukainen et al. 2013). Vg is considered an important part of honey bee health, survival, and lifespan; it improves the nutritional status of bees, protecting them from oxidative and immune attacks (Amdam and Omholt 2002; Havukainen et al. 2013). Chlorella provides a protein-rich diet, which led to elevated $\mathrm{Vg}$ transcript levels. It is known that $\mathrm{Vg}$ inhibits the $\mathrm{JH}$ production, which acts as a pro-ageing factor in bees (Libbrecht et al. 2013). We did not observe any effect of Chlorella on the $\mathrm{JH}$ pathway from $J H E$ transcript levels, but Chlorella decreased TOR and InR2 transcript levels. TOR is a conserved Ser/Thr kinase that controls organismal growth and metabolism in response to nutrient availability. In adulthood, partial inhibition of $T O R$ function leads to prolonged lifespan, possibly by mimicking calorific restriction (Antikainen et al. 2017; Stanfel and Shamieh 2009). Similarly, numerous studies on insulin signalling pathways in both vertebrates and invertebrates show that it influences the ageing process and its inhibition extends lifespan and delays agerelated disease (Altintas et al. 2016; Bartke 2008; Kenyon 2011).

In summary, we found that Chlorella had positive effects on bee colony development, via beneficial changes in metabolism, including increased fat deposition and Vg transcript levels, and decreased TOR and InR2 transcript levels. Our results suggest that the nutritional composition of Chlorella might be an appropriate food supplement for honey bees.

\section{ACKNOWLEDGEMENTS}

The authors thank Mrs. H. Štěrbová for her technical assistance. The English grammar and stylistics were checked by the Editage Author Services. 


\section{AUTHORS' CONTRIBUTION}

RČF, DK, and TJ conceived this research and designed experiments; $\mathrm{TJ}$ participated in the design and interpretation of the data; TJ, JK, MS, AT, VK, and JD performed experiments and analysis; RČF and DK wrote the paper and participated in the revisions of it. All authors read and approved the final manuscript.Funding information

This study was supported by grant no. LTAUSA17116 Inter Action from Ministry of Education of the Czech Republic, by project Strategy AV21, Diversity of Life and Health of Ecosystems.

\section{COMPLIANCE WITH ETHICAL STANDARDS}

Conflict of interest The authors declare that they have no conflict of interest.

Effets de la Chlorella sp. sur les caractéristiques biologiques de l'abeille Apis mellifera

Apis mellifera / Chlorella / nutriments / longévité

Effekte von Chlorella sp. auf biologische Charakteristika der Honigbiene Apis mellifera

Apis mellifera / Chlorella / Lebensdauer / Nährstoffe

\section{REFERENCES}

Altintas, O., Park, S., Lee, S. V. (2016) The role of insulin/ IGF-1 signaling in the longevity of model invertebrates, C. elegans and D. melanogaster. BMB Rep. 49, 81-92

Amdam, G.V., Omholt, S.W. (2002) The regulatory anatomy of honeybee lifespan. J. Theor. Biol. 216, 209-228

Antikainen, H., Driscoll, M., Haspel, G., Dobrowolski, R. (2017) TOR-mediated regulation of metabolism in aging. Aging Cell 16, 1219-1233

Arrese, E.L. and Wells, M.A. (1994) Purification and properties of a phosphorylatable triacylglycerol lipase from the fat body of an insect, Manduca sexta. J Lipid Res 35, 1652-1660.
Aurori, C.M., Buttstedt, A., Dezmirean, D.S., Liviu, A.M., Moritz, R.F.A., Erler, S. (2014) What is the main friver of sgeing in long-lived winter honeybees: antioxidant enzymes, innate immunity, or vitellogenin? J. Gerontol. Biol. Sci. 69, 633-639

Avni, D., Hendriksma, H.P., Dag, A., Uni, Z., Shafir, S. (2014) Nutritional aspects of honey bee-collected pollen and constraints on colony development in the eastern Mediterranean. J. Insect Physiol. 69, 65-73

Bartke, A., 2008. Insulin and aging. Cell Cycle 7, 33383343. doi:https://doi.org/10.4161/cc.7.21.7012

Brodschneider, R., et al. (2018) Multi-country loss rates of honey bee colonies during winter 2016/2017 from the COLOSS survey. J. Apic. Res. 57, 452-457

DeGrandi-Hoffman, G., Chen, Y., Huang, E., Huang, M.H. (2010). The effect of diet on protein concentration, hypopharyngeal gland development and virus load in worker honey bees (Apis mellifera L.). J. Insect Physiol. 56, 1184-1191

DeGrandi-Hoffman, G., Gage, S.L., Corby-Harris, V., Carroll, M., Chambers, et al. (2018). Connecting the nutrient composition of seasonal pollens with changing nutritional needs of honey bee (Apis mellifera L.) colonies. J. Insect Physiol. 109, 114-124

Di Pasquale, G., Salignon, M., Le Conte, Y., Belzunces, L.P., Decourtye, A., et al. (2013) Influence of pollen nutrition on honey bee health: Do pollen quality and diversity matter? PLoS One 8, 1-13

Downer R.G.H. (1985). Lipid metabolism. Pp. 77-113 in G.A.Kerkut and L.I. Gilbert, eds. Comprehensive Insect Bio-chemistry, Physiology and Pharmacology. Vol. 10. Pergamon, Oxford.

Eremia, N., Zagareanu, A., Mardari, T., Modvala, S. (2013) Stimulation of Resistance of Bee Families during Wintering. Anim. Sci. Biotechnol. 46, 268-271

Havukainen, H., Munch, D., Baumann, A., Zhong, S., Halskau, O., et al. (2013) Vitellogenin recognizes cell damage through membrane binding and shields living cells from reactive oxygen. J. Biol. Chem. 288, 28369-28381

Hsieh, Y.S., Hsu, C.Y. (2011) The changes of age-related molecules in the trophocytes and fat cells of queen honeybees (Apis mellifera). Apidologie 42, 728-739

Hsu, C., Chuang, Y., Chan, Y. (2014) Changes in cellular degradation activity in young and old worker honeybees (Apis mellifera). EXG 50, 128-136

Kenyon, C. (2011) The first long-lived mutants: discovery of the insulin/IGF-1 pathway for ageing. Philos. Trans. R. Soc. B Biol. Sci. 366, 9-16

Kodrík, D., Socha, R., Šimek, P., Zemek, R., Goldsworthy, G.J. (2000) A new member of the AKH/RPCH family that stimulates locomotory activity in the firebug, Pyrrhocoris apterus (Heteroptera). Insect Biochem. Mol. Biol. 30 , 489-498

Kodrík D. (2008) Adipokinetic hormone functions that are not associated with insect flight. Physiol. Entomol. 33, 171-180. 
Kodrík, D., Vinokurov, K., Tomčala, A., Socha, R. (2012) The effect of adipokinetic hormone on midgut characteristics in Pyrrhocoris apterus L. (Heteroptera). J. Insect Physiol. 58, 194-204

Kodrík D., Bednářová D., Zemanová Z., Krishnan N., (2015) Hormonal Regulation of Response to Oxidative Stress in Insects - An Update. International Journal of Molecular Sciences 16(10), 25788-25816

Lorenz M.W., Kellner R., Woodring J., Hoffmann K.H., Gäde G., (1999) Hypertrehalosaemic peptides in the honeybee (Apis mellifera): purification, identification and function. Journal of Insect Physiology 45 (7), 647653

Lorenz, M.W. (2001). Synthesis of lipid in the fat body of Gryllus bimaculatus: age-dependency and regulation by adipokinetic hormone. Arch Insect Biochem Physiol. 47, 198-214

Libbrecht, R., Corona, M., Wende, F., Azevedo, D.O., Serrao, J.E., Keller, L. (2013). Interplay between insulin signaling, juvenile hormone, and vitellogenin regulates maternal effects on polyphenism in ants. Proc. Natl. Acad. Sci. 110, 11050-11055

Marchal, E., Schellens, S., Monjon, E., Bruyninckx, E., Marco, H.G. et al. (2018). Analysis of peptide ligand specificity of different insect adipokinetic hormone receptors. Int. J. Mol. Sci. 19

Münch, D., Amdam, G. V. (2010) The curious case of aging plasticity in honey bees. FEBS Lett. 584, 2496-2503

Mutti, N.S., Dolezal, A.G., Wolschin, F., Mutti, J.S., Gill, K.S., Amdam, G. V. (2011). IRS and TOR nutrientsignaling pathways act via juvenile hormone to influence honey bee caste fate. J. Exp. Biol. 214, 39773984

Naug, D., Gibbs, A. (2009) Behavioral changes mediated by hunger in honeybees infected with Nosema ceranae. Apidologie 40, 595-599

Neumann, P., Carreck, N.L. (2010). Honey bee colony losses. J. Apic. Res. 49, 1-6

Omar, E., Abd-Ella, A.A., Khodairy, M.M., Moosbeckhofer, R., Crailsheim, K., Brodschneider, R.(2017) Influence of different pollen diets on the development of hypopharyngeal glands and size of acid gland sacs in caged honey bees (Apis mellifera). Apidologie 48, 425-436

Patel, A., Fondrk, M.K., Kaftanoglu, O., Emore, C., Hunt, G., Frederick, K., Amdam, G. V. (2007) The making of a queen: TOR pathway is a key player in diphenic caste development. PLoS One 1-7.

Peng, Y., D’Antuono, M., Manning, R. (2012) Effects of pollen and artificial diets on the hypopharyngeal glands of newly hatched bees (Apis mellifera L.). J. Apic. Res. 51, 53-62

Potts, S.G., Biesmeijer, J.C., Kremen, C., Neumann, P., Schweiger, O., et al. (2010) Global pollinator declines: Trends, impacts and drivers. Trends Ecol. Evol. 25, 345-353

Schneedorferová, I., Tomčala, A., Valterová, I. (2015) Effect of heat treatment on the n-3/n- 6 ratio and content of polyunsaturated fatty acids in fish tissues. Food Chem. 176, 205-211

Schneider, M., Dorn, A. (1994) Lipid storage and mobilization by flight in relation to phase and age of Schistocerca gregaria females. Insect Biochem. Mol. Biol. 24, 883-889

Socha, R., Kodrík, D., Šimek, P., Patočková, M. (2004) The kind of AKH-mobilized energy substrates in insects can be predicted without a knowledge of the hormone structure. Eur. J. Entomol. 101 , 1210-5759

Standifer, L.N., Moeller, F.E., Kauffeld, N.M., Herbert, E. W. J, Shimanuki, H. (1978). Supplemental feeding of honey bee colonies. United States Dep. Agric. Agric. Inf. Bull. 413, 1-8

Stanfel, M.N., Shamieh, L.S., Kaeberlein, M., K, K.B. (2009). The TOR pathway comes of age. Biochim. Biophys. Acta 1790, 1067-1074

Stoscheck, C.M. (1990) Quantitation of protein. Methods Enzymol. 182, 50-68

Veenstra, J.A., Rodriguez, L., Weaver, R.J. (2012) Allatotropin, leucokinin and $\mathrm{AKH}$ in honey bees and other Hymenoptera. Peptides 35, 122-130

Wang, H., Zhang, S., Zeng, Z., Yan, W., Wang, H.et al. (2014). Nutrition affects longevity and gene expression in honey bee (Apis mellifera) workers. Apidologie 45, 618-625

Winston, M.L. (1987). The Biology of the Honey Bee. Harward University Press, Cambridge, Massachusetts

Publisher's note Springer Nature remains neutral with regard to jurisdictional claims in published maps and institutional affiliations. 